\title{
HIGH RESOLUTION WAVE TANK TESTING OF SCALED WAVE ENERGY DEVICES
}

\author{
Ken Rhinefrank, Al Schacher, Joe Prudell \\ Columbia Power Technologies, LLC \\ 3079 Kelley Engineering Center \\ Corvallis, Oregon 97331
}

\author{
Chad Stillinger, David Naviaux, \\ Ted Brekken, Annette von Jouanne \\ School of Electrical Engineering and Computer Sci. \\ Oregon State University \\ Corvallis, Oregon 97331
}

\author{
David Newborn, Solomon Yim, Dan Cox \\ O. H. Hinsdale Wave Research Laboratory \\ Oregon State University \\ Corvallis, Oregon 97331
}

\begin{abstract}
In many industries, such as the wave energy industry, the importance of accurate physical model testing in the development process to full scale devices cannot be overemphasized. This paper presents a new, high-precision wave tank testing system and process designed and implemented by Columbia Power Technologies (CPT) and Oregon State University (OSU). The system's high level of functionality was demonstrated during characterization of CPT's wave energy converter (WEC) and is now established at OSU's O.H. Hinsdale Wave Research Laboratory (HWRL), in collaboration with the Northwest National Marine Renewable Energy Center (NNMREC) headquartered at OSU. The critical instrumentation, optical motion tracking system, data acquisition and related components developed for wave tank testing are fully characterized herein, and the paper concludes with example testing of a scaled wave energy device including experimental results.
\end{abstract}

\subsection{INTRODUCTION}

Experimental hydrodynamic analysis provides a cost effective solution to modeling complicated fluid forcing on ocean structures. In broad terms, scaled experimental hydrodynamics retain the complicated hydraulic processes that exist around the system of interest, allow for cheaper and easier quantifiable measurements of relevant criteria or parameters, and permits researchers to accurately control incident fluid forcing on models [1]. Also, conditions that are difficult to evaluate analytically or numerically can be included in experimental tests, such as spread spectrum irregular seas, extreme wave heights and slopes, or survivability conditions corresponding to long return period sea states.

Scale models of systems such as ships, submarines, and buoys allow researchers to evaluate dynamic responses in regular and irregular seaways. Incident regular waves on scale models can be used to verify the accuracy of linear, frequency- domain numerical analysis. Irregular wave conditions force the scale model in a similar manner as a seaway that may be encountered in the ocean, and can be used to compare timedomain simulations.

Scale experimental hydrodynamic testing of wave energy converters (WECs) requires special attention to particular aspects of the system that is being modeled. As with all scaled hydrodynamic analysis, scale effects due to viscous boundary layers and incompressible flow regions should be minimized. In addition, accurate modeling of a WEC power takeoff (PTO) system affects the coupled hydrodynamic and power generation responses of the system. An outline of other considerations for scaled WEC laboratory testing is provided in [2].

\subsection{HIGH-PRECISION WAVE TANK SYSTEM}

OSU's O.H. Hinsdale Wave Research Laboratory (HWRL) operates one of the largest coastal and ocean hydraulic laboratories in North America including: a three-dimensional wave basin with large-stroke, multi-directional wavemaker (Fig. 1) and a large wave flume (104m long) with a new, highperformance, large-stroke piston-type wavemaker. Collaboration with Columbia Power Technologies (CPT) has enabled a high-precision wave tank testing system and process to be established in conjunction with the Northwest National Marine Renewable Energy Center (NNMREC) headquartered at OSU, to facilitate the optimization of wave energy devices to efficiently convert the motion of ocean waves into electrical energy.

NNMREC is a partnership between OSU (wave), the University of Washington (UW, tidal) and the National Renewable Energy Laboratory (NREL). The mission of NNMREC is to facilitate commercialization of marine energy technology, inform regulatory and policy decisions, and to close key gaps in scientific understanding. Primary center activities include: 1 ) development of facilities to serve as an 
integrated, standardized test Center for U.S. and international developers of wave and tidal energy; 2) evaluation of potential environmental, ecosystem and social impacts, focusing on the compatibility of marine energy technologies in areas with sensitive environments and existing users; 3) device and array optimization for effective deployment of wave and tidal energy technologies; and 4) increased reliability and survivability of marine energy systems.

\subsection{TESTING SYSTEM}

In order to reliably test and accurately characterize the scaled hydrodynamic model of a wave energy buoy, a high-precision wave tank testing system was developed within the existing HWRL. . Three separate data acquisition systems (the HWRL data acquisition system, the dSPACE WEC data acquisition system, and the PhaseSpace optical motion tracking system described in Section 2.3) were synchronized using a start and stop TTL trigger corresponding with the wavemaker start-stop signal. The directional basin, named the tsunami wave basin (TWB), is $48.8 \mathrm{~m} \mathrm{~L} \times 26.5 \mathrm{~m} \mathrm{~W} \times 2.1 \mathrm{~m} \mathrm{D}$. The maximum wave heights are $0.8 \mathrm{~m}$ in $1 \mathrm{~m}$ water depth, with periods $(\mathrm{T})$ of 0.5-10s. The integrated components for this experiment included wave gauges, wave and current profilers, onboard sensors, DC motor-generator damping, dSPACE data acquisition and control, and PhaseSpace motion tracking cameras and LED markers.

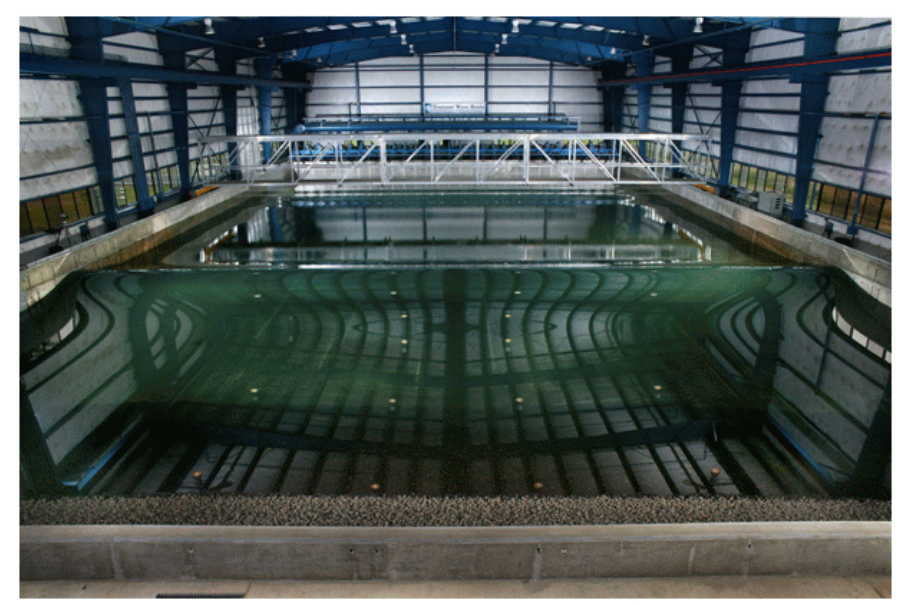

Fig. 1. Directional wave basin at Oregon State University.

\subsection{PROCESS}

For the wave tank testing, an integrated system approach was implemented to ensure that calibrated data sets were recorded in a well organized and synchronized format for each specific test. The integrated operation of the wavemaker, wave gauges, wave and current profilers, onboard sensors, DC motor-generator damping, dSPACE data acquisition and control, and PhaseSpace motion tracking cameras and LED markers, were operated by three researchers and overseen by a lead test coordinator. Each test day was initiated by a PhaseSpace motion tracking system calibration and alignment to ensure the WEC was accurately tracked with six degrees of freedom for each rigid body. The WEC was then connected, followed by the mooring load cell calibration check, WEC damping linearity check, and WEC zero point station alignment check. A still water wave gauge calibration check was performed and a calibration wave was run by HWRL staff. Hand held radios provided communication between system stations.

For each wave set, the data was recorded with a specific code and number related to the test. All the data sets were post processed to $50 \mathrm{~Hz}$ and aligned with the trigger signal. Scaled monochromatic wave sets of varying frequency and amplitude, as well as stochastic real sea wave profiles for Newport, OR, Lewis UK, and Orkney UK, with varying spectral shapes and spreading functions, were run in the wave basin which provided discrete WEC data point hydrodynamic model validation. Amplitude scans included wave heights of $1 \mathrm{~m}, 2 \mathrm{~m}$ and $3 \mathrm{~m}$ full scale equivalent and were performed to evaluate the affect of wave amplitude on the response amplitude operators (RAO's); this verified test conditions were consistent with linear wave theory assumptions and confirmed that $2 \mathrm{~m}$ wave heights were appropriate for the remaining frequency domain scans. Response amplitude operators (RAO's) were discretely graphed from each of the frequency and amplitude scans using the motion tracking system data, and the relative capture widths (RCWs) were plotted using the torque sensor and encoder data from dSPACE. The high resolution of the calibrated motion tracking system was validation for the onboard WEC accelerometer and encoder, and also provided redundancy confirmation in the data collection. Separate power supplies and isolated data acquisition cables ensured reliable quality data recording. The onboard WEC sensors, circuits, and data collection components were incorporated into the scaled model design for the correct scaled center of gravity (CG), moments of inertia, and total mass of the system per the designed waterline.

\subsection{OPTICAL MOTION TRACKING SYSTEM}

To track the buoy in the tank accurately, a PhaseSpace optical motion tracking system was procured and installed in OSU's HWRL. This system is a third party product developed for the entertainment industry, and this work is the first application of PhaseSpace to quantify the response of floating structures to wave action. The optical motion tracking system includes active markers that are placed strategically on various moving or fixed test bodies in the wave tank, along with cameras to track the motion of those components. During wave action, the system of markers and cameras allows the motion of the marked components to be tracked and recorded with submillimeter resolution at a $480 \mathrm{~Hz}$ sample rate. 


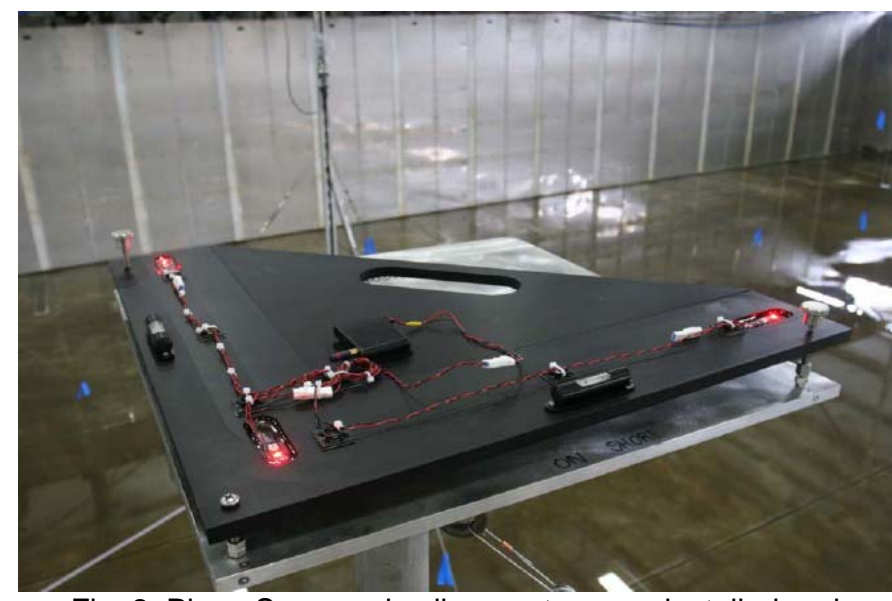

Fig. 2. PhaseSpace axis alignment square installed and leveled in the wave basin.

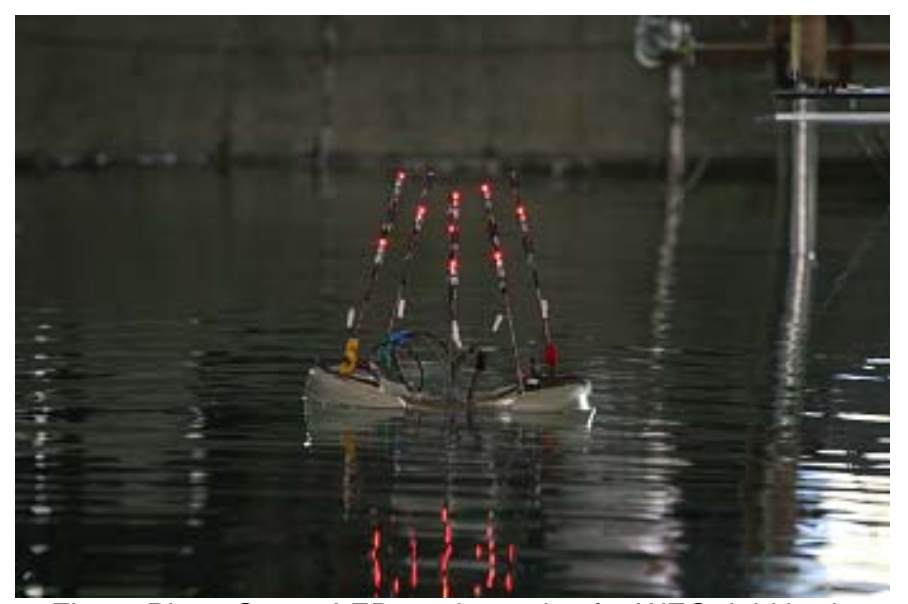

Fig. 3. PhaseSpace LED marker poles for WEC rigid body tracking in the wave basin.

The motion capture system characterized for this test (Fig. 2) provides 6-sigma position accuracy of $0.9 \mathrm{~mm}$ for all targets within a $1.2 \mathrm{~m}$ radius, and $1.3 \mathrm{~mm}$ accuracy up to a $2.5 \mathrm{~m}$ radius. The axis alignment square in Fig. 2 is aligned normal to the incoming wave and leveled to provide proper orientation of the motion capture system. Motion data is recorded at $480 \mathrm{~Hz}$ and down-sampled to $50 \mathrm{~Hz}$ for model evaluation. The 18-LED's shown in Fig. 3 are digitally identified and assigned to each body. LED's were viewed by eight cameras located $3.05 \mathrm{~m}$ above the water on a $6.1 \mathrm{~m}$ by $6.1 \mathrm{~m}$ frame.

\subsection{DATA ACQUISITION SYSTEM}

To monitor and record the data required for analysis, experimental data from three separate sources was captured; HWRL wave height data, PhaseSpace optical motion tracking system data, and the WEC internal operating parameters.

During each test, the wave height data was captured to a PC located in the HWRL control room. An onshore PC, located approximately $20 \mathrm{~m}$ from the WEC under test, was used to capture the data reported by the optical motion tracking system. A dSPACE single board rapid prototyping system (DS1103), located at a second onshore PC, allowed power take-off (PTO) optimization and data collection of internal WEC operating parameters. An embedded proprietary control algorithm allowed the internal operating parameters of the WEC to be monitored via an RS-422 sub-marine communication cable connected to the WEC.

The internal operating parameters of the WEC were reported to the DS1103 every 2ms during testing by a custom designed embedded controller (EC) located in the nacelle of the WEC. A six-degrees-of-freedom inertial sensor, integrated into the EC, was used to monitor the linear acceleration and angular velocity for each of the 3-axes of motion. The EC also monitored the high-resolution encoder attached to each of the two rotary generators. The output of each encoder was processed by the EC to accurately determine the angular position and velocity of each generator shaft relative to the WEC spar. In addition, the EC measured the torque applied to each float drive shaft via two torque transducers integrated into the WEC. The combination of float torque and generator speed allowed for precise measurement of mechanical shaft power absorbed by the WEC (the figure of merit used to compute RCW).

A custom interface programmed with dSPACE's ControlDesk acquisition and control environment allowed an operator to view the current testing parameters in real-time as the various tests were being performed. Section 5.0 presents example testing.

\subsection{EXAMPLE WEC SYSTEM UNDER TEST}

CPT's wave energy converter (WEC) tested during this work was a point absorber designed to convert both heave and surge wave energy directly into rotary motion to harness twice the energy of other point absorbers operating solely in heave [3]. CPT's WEC, shown schematically in Fig. 4, is a Direct Drive Rotary (DDR) system comprised of three moving bodies: a forward float, aft float, and spar [4]. Each float was attached to the spar through a sealed high torque drive shaft. The forward float was attached to the starboard drive shaft and DDR generator, and the aft float was connected to the port. The bottom of the spar was attached to a large damper tank and is designed to stay relatively stationary in the heave direction. The floats had a $90^{\circ}$ range of motion centered about the horizontal axis. In action, the spar moved forward to aft and the floats heaved and surged out of phase with each other in a cyclic motion as the waves passed.

During this work, a 1:33 scale model was experimentally tested (Fig. 3 ) in collaboration with Garrad Hassan and OSU. The test buoy is a 1:33 Froude scaled replica (kinematic similitude of 1:5.74) of the full scale system. The buoy was built in collaboration with Ershigs Inc. (composites 
manufacturing partner) to within $1 \%$ of the full scale prototype.

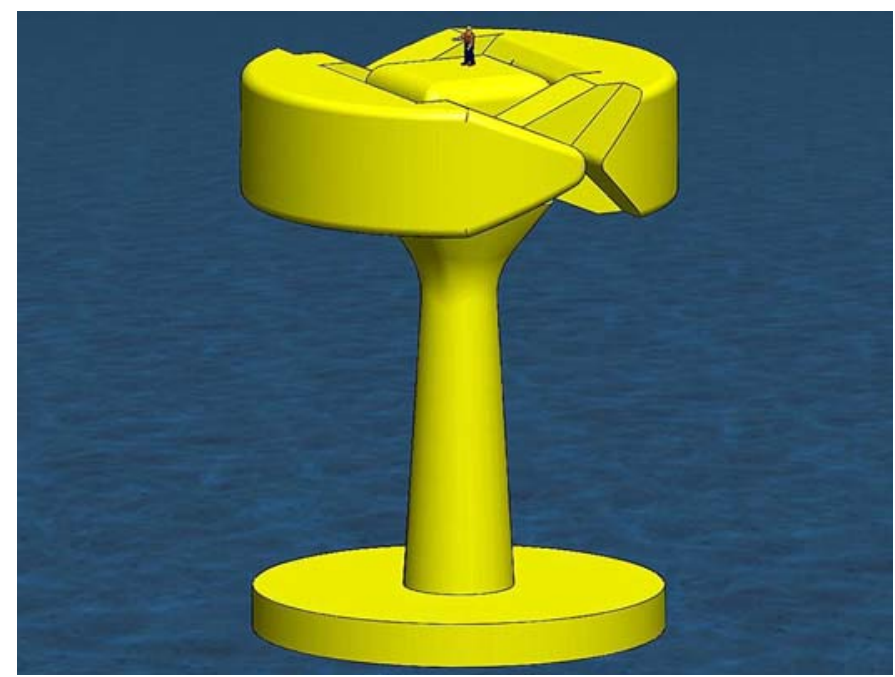

Fig. 4. Columbia Power's direct drive rotary WEC.

\subsection{CONTROL MODES FOR TESTING}

The onboard data acquisition module digitally sent PTO speed and generator torque data from the encoders and the torque transducers to dSPACE. dSPACE used a Matlab/Simulink add-on to provide a powerful and flexible control platform that was easily modified to achieve a wide variety of control approaches (Fig. 5). dSPACE then commanded the HiRel servo control board to update the motor current to achieve the desired torque. These systems worked together to allow precise control of the WEC PTO damping. This high speed $500 \mathrm{~Hz}$ (2ms) dynamic control loop allowed for any real-time PTO damping up to the torque rating of the scaled PTO.

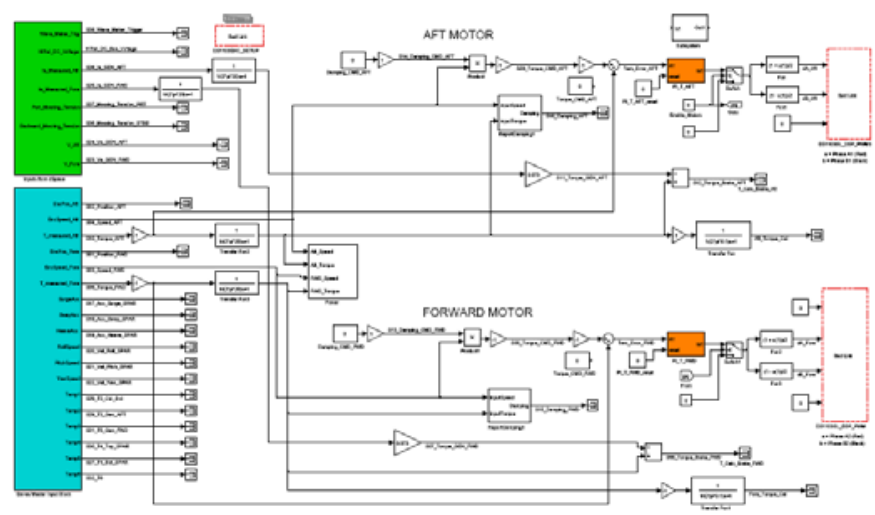

Fig. 5. Schematic of Simulink control approach used by dSPACE.

A strict weight constraint was put on the scaled PTO to guarantee an exact replica of the full scale WEC. The largest servo motors that fell within this weight constraint had insufficient torque for this application. An innovative arrangement of lightweight passive hydraulic dampers and actively controlled servo motors allowed for a light, strong, and accurate PTO solution. The passive damping elements were adjusted to provide a baseline torque to approximate the desired damping characteristics. The torque transducer on each PTO measured the error between the passive element torque and the desired torque set point. The servo motors then assisted the damper torque with excellent precision to accomplish the exact torque specification in real time.

A wide range of time domain torque profiles were possible with this PTO design including both real and reactive controls. In addition to evaluating the design performance of the WEC, the scaled tank test data were used to validate both time- and frequency-domain numerical models. The frequency domain model under consideration was limited to a velocity proportional (linear) damping. Then this rather simple linear control mode was able to be used in combination with the observed WEC motion. The damping set point was computed by multiplying the current PTO speed with the damping coefficient in units of "Nms". The damping coefficient was adjusted between trials to target the best absorption for a given wave climate. Future testing will broaden the time domain control approach to include maximum power point tracking, best continuous power, and reactive control.

\subsection{EXAMPLE TESTING}

With three synchronized data collection systems, over 80 unique parameters were stored. Parameters critical to the test were displayed in real time using the graphical user interface (GUI) of dSPACE (Fig. 6). This allowed researchers to observe testing conditions and provided real time diagnostics.

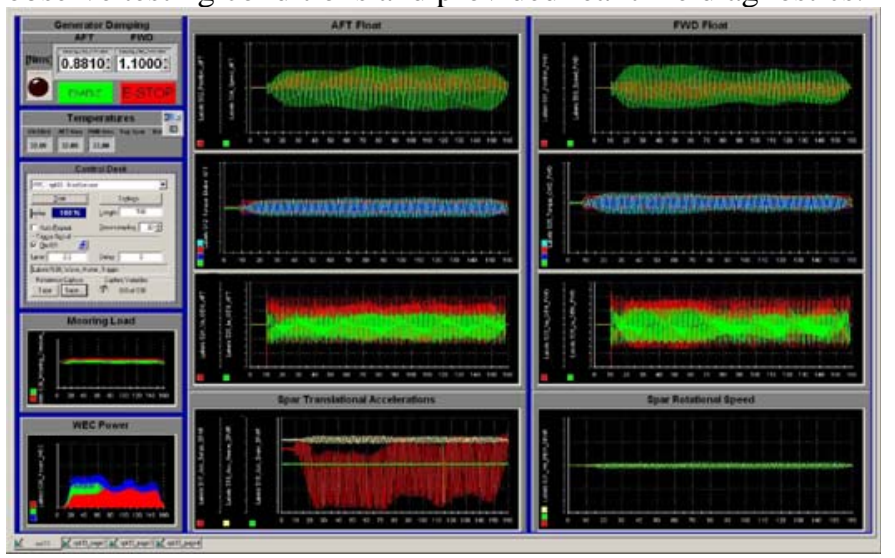

Fig. 6. Screen shot of dSPACE GUI during testing.

PhaseSpace provided the absolute position in 6 degrees for each of the three bodies of the WEC with high precision. This information is invaluable for numerical model validation. However, in full-scale ocean tests it may be impractical to employ a PhaseSpace type of motion tracking system. To overcome this deficiency and to bridge the gap between controlled tank testing and at-sea trials, redundancy was designed into the motion capture hardware. PhaseSpace was used to validate the motion data provided by the onboard 
accelerometer and encoders, components which are more readily included on an ocean test specimen. Fig. 7 shows the time series of the PhaseSpace PTO positions and the encoder PTO positions. Fig. 8 is a magnified section of Fig.7 to show the excellent agreement between PhaseSpace computed position (heavy line) and encoder position (light line).

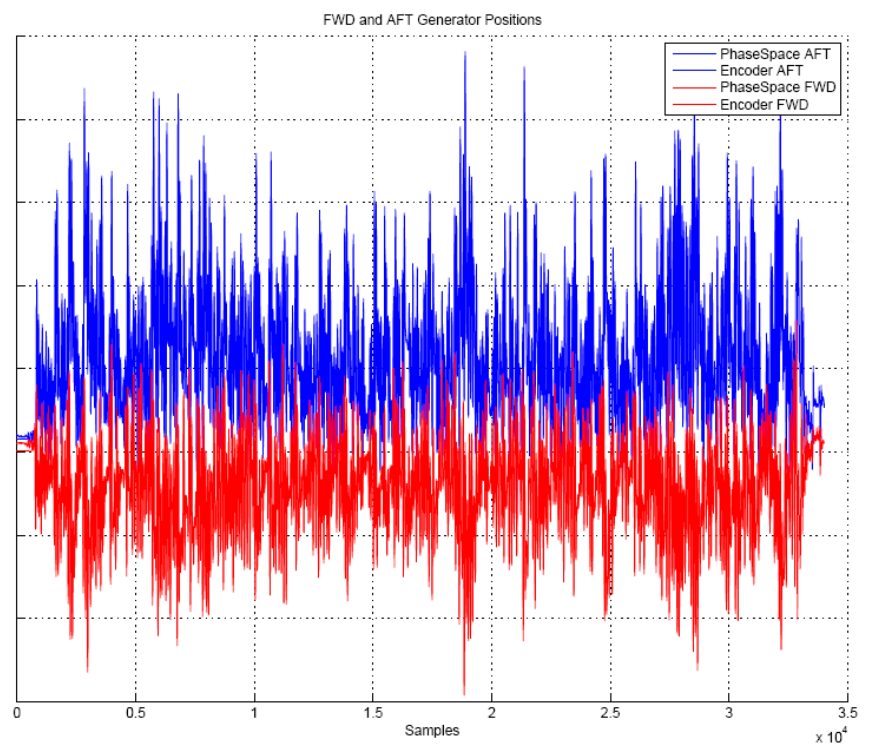

Fig. 7. Time series of the PTO rotation for forward and aft floats for both Phasespace and the Encoders.

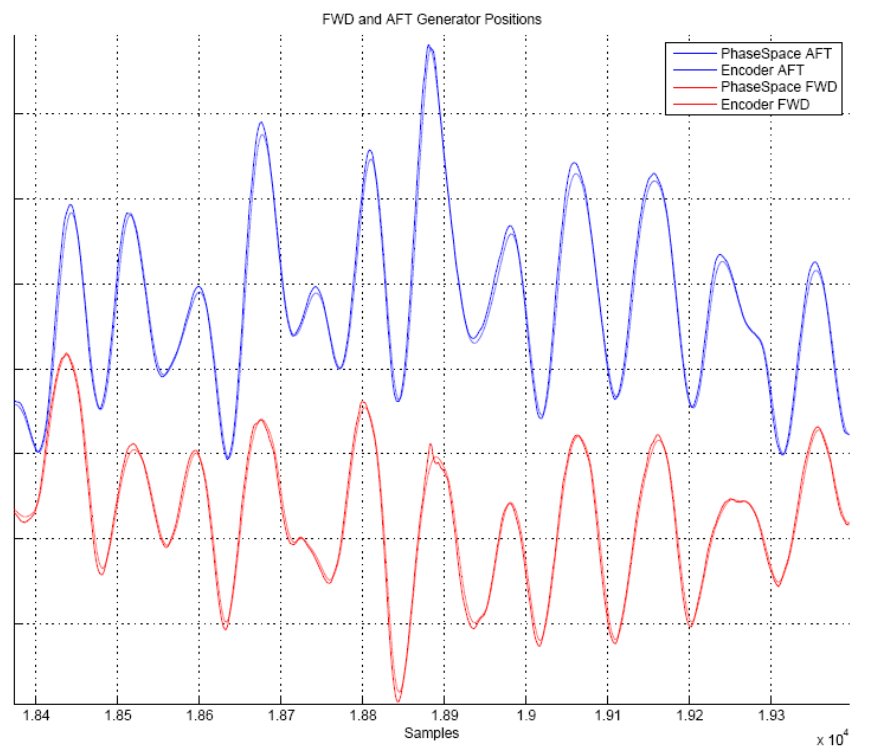

Fig. 8. Close up of PTO position time series.

\subsection{CONCLUSIONS}

This paper presented a new, high resolution wave tank testing system and process for wave energy converters (WECs) to validate hydrodynamic models and to provide indications of offshore device behavior. This testing system and processes were designed and implemented by Columbia Power Technologies (CPT) and Oregon State University (OSU), in OSU's O.H. Hinsdale Wave Research Laboratory (HWRL) in collaboration with the Northwest National Marine Renewable Energy Center (NNMREC) headquartered at OSU. The paper describes the integration of the critical instrumentation including wave gauges, wave and current profilers, onboard sensors, a dSPACE data acquisition and control system, and PhaseSpace motion tracking cameras and LED markers. During wave action, the system of markers and cameras allowed the motion of the marked wave energy buoy components to be tracked and recorded with sub-millimeter resolution at a $480 \mathrm{~Hz}$ sample rate. A system for highly accurate control of PTO damping on a small scale WEC was also demonstrated. Finally, example wave tank testing results were presented from the successful testing of CPT's 1:33 scale WEC.

\subsection{ACKNOWLEDGEMENTS}

The authors recognize the Columbia Power board of directors for their continued pursuit of clean renewable Power and their investment in ocean wave energy. The support of the US Navy (NAVFAC) and the Oregon delegation has been a tremendous benefit to this project. We also acknowledge the fine work performed by Garrad Hassan, Crescere Marine Engineering, and Ershigs Inc.

The HWRL is partially supported by the George E. Brown, Jr. Network for Earthquake Engineering Simulation (NEES) Program of the National Science Foundation under Award Number CMMI-0402490. The PhaseSpace motion capture system was purchased through OSU's Research Equipment Reserve Fund with additional funding support from Pacific Power and CPT, including an educational discount from PhaseSpace. The technical support of Linda Fayler, Jason Killian, Tim Maddux, and Sungwon Shin at the HWRL is also greatly appreciated.

\subsection{REFERENCES}

[1] S. A. Hughes, "Physical Models and Laboratory Techniques in Coastal Engineering", World Scientific Advanced Series on Ocean Engineering - Volume 7, 1993, ISBN 981-02-1540-1.

[2] J. Cruz, ed. “Ocean Wave Energy: Current Status and Future Prespectives", Springer Series in Green Energy and Technology, 2008, ISBN 978-3-540-74894-6.

[3] K. Budal, J. Falnes, "A resonant point absorber of ocean-wave power", Nature, vol. 256, Aug. 7, 1975, p. 478, 479.

[4] K. Rhinefrank, J. Prudell, A. Schacher, "Development and Characterization of a Novel Direct Drive Rotary Wave Energy Point Absorber”, MTS-IEEE Oceans Conference Proceedings, 2009. 